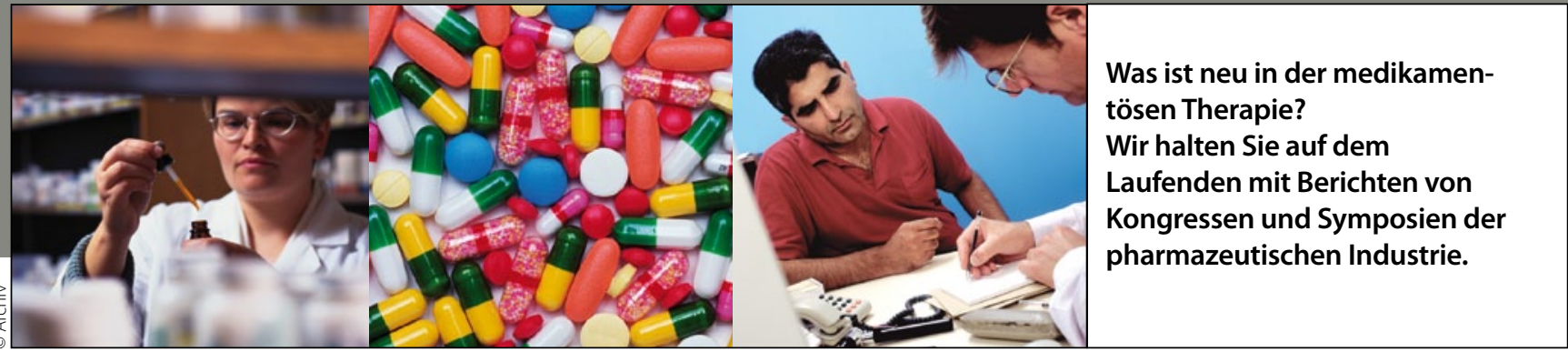

\title{
Ejaculatio praecox Bedarfstherapie bringt Harmonie zurück ins Schlafzimmer
}

- Die Ejaculatio paecox (EP) ist eine männliche Sexualstörung, die mit einer Prävalenz von bis zu $20 \%$ wahrscheinlich deutlich häufiger ist als die erektile Dysfunktion (ED). Man unterscheidet angeborene (ca. 75\%) und erworbene Varianten (ca. 25\%). Der primären Form liegt eine Störung des Serotoninstoffwechsels im Sinne einer Hypofunktion des $5-\mathrm{HT}_{2 \mathrm{c}}$-Rezeptors und/oder $\mathrm{Hy}$ perfunktion des $5-\mathrm{HT}_{1 \mathrm{a}}$-Rezeptors zugrunde, berichtete der Urologe und Sexualmediziner Prof. Hartmut Porst, niedergelassen in Hamburg. Die extremste Form ist die Ejaculation ante portas.

Charakterisiert ist die Störung durch eine vom Patienten nicht kontrollierbare frühe Ejakulation, die meist innerhalb von einer Minute nach der Penetration erfolgt. Diese sog. „intravaginale Ejakulationslatenzzeit" (IELT) liegt bei gesunden Männern in der Regel im Bereich von 3-15 Minuten, so Porst. Weiterhin gehören zur Krankheitsdefinition negative persönliche Folgen, wie Leidensdruck, Frustration über ein unerfülltes Sexualleben, Vermeidung sexueller Aktivität und Partnerprobleme.

\section{„Die Frauen bleiben auf dem Trockenen"}

Porst berichtete über Patienten, die nach frustrierenden Partnerschaften und vergeblichen Selbsttherapieversuchen das Problem mit reichlich Sport und beruflichen Karrieren sublimieren. Am meisten leiden die Partnerinnen, so Porst. Der Patient selbst sucht selten ärztliche Hilfe, die Schambarriere scheint noch höher als bei der erektilen Dysfunktion. Auch Ärzte scheuen offenbar das Gespräch über Sexualität. Die gute Behandelbarkeit der EP hat sich noch nicht herumgesprochen.

\section{Therapieoptionen}

Therapieoptionen sind die Sexual- und Paartherapie sowie eine pharmakologische Behandlung, erklärte der in Freiburg niedergelassene Urologe Dr. Thomas Kreutzig. Sexualtherapeutisch werden die StoppStart-Technik sowie die Squeeze-Technik angewandt. Medikamentös kommen beschichtete Kondome, off label kommen Lokalanästhetika, Tramadol oder langwirksame Serotonin-Wiederaufnahme-Hemmer (SSRI) in Betracht.

Zugelassen in der Indikation ist der kurzwirksame SSRI Dapoxetin (Priligy ${ }^{\circledR}$ ), der in zwei Dosierungen (30 oder 60 mg) zur Verfügung steht. Er flutet schnell an und $\mathrm{ab}$ und wird bei Bedarf eingenommen.
Die Verträglichkeit ist besser als die einer Dauertherapie mit langwirksamen SSRI. Die häufigsten dosisabhängigen Nebenwirkungen sind Kopfschmerzen, Schwindel und Übelkeit.

Die Wirksamkeit wurde in fünf klinischen Studien bei über 6000 Patienten getestet, schilderte Kreutzig. Wesentliche Ergebnisse: Dapoxetin verdreifacht im Mittel die IELT von ca. einer auf drei bis vier Minuten. Dadurch erreichen etwa 40\% der Patienten eine hohe bis sehr hohe Zufriedenheit mit dem Sexualleben. Der Therapieerfolg sollte aber unbedingt nach vier Wochen oder sechs Dosen vom Arzt kontrolliert werden, so Kreutzig.

\section{- Dr. med. Dirk Einecke}

Quellen:„Ejaculatio praecox: 1, 2, 3... schon vorbei!", Symposium beim Internistenkongress, Wiesbaden, April 2013 (Veranstalter: Berlin Chemie).

\section{Kurz notiert}

Chronisch entzündliche Darmerkrankung oder Reizdarm? - Um hier Klarheit zu schaffen eignet sich der Schnelltest PreventID ${ }^{\otimes}$ CalScreen $^{\circledR}$ mit einem Schwellenwert von 50 $\mu \mathrm{g} / \mathrm{g}$. Ein weiterer Calprotectin-Schnelltest, der PreventID ${ }^{\circledR}$

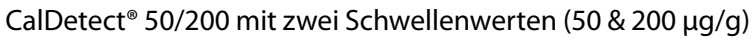
ermöglicht neben dem Screening auch die individuelle Therapiekontrolle von CED-Patienten. Der aktuelle Patientenflyer erklärt die Rolle von Calprotectin bei der Abklärung von Darmentzündungen sowie den Einsatz der Schnellteste verständlich und übersichtlich. Das Faltblatt sowie weiteres Informationsmaterial für medizinisches Fachpersonal können gratis bei Preventis unter der Telefonnummer 06251/70 7110 oder unter info@preventis-online.de angefordert werden.

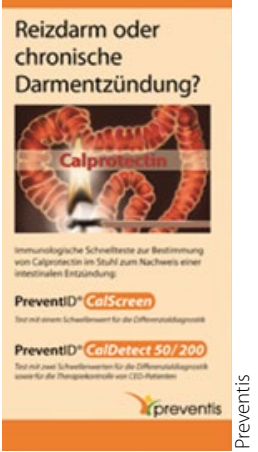

